\title{
Validity and reliability of the WIMU inertial device for the assessment of the vertical jump
}

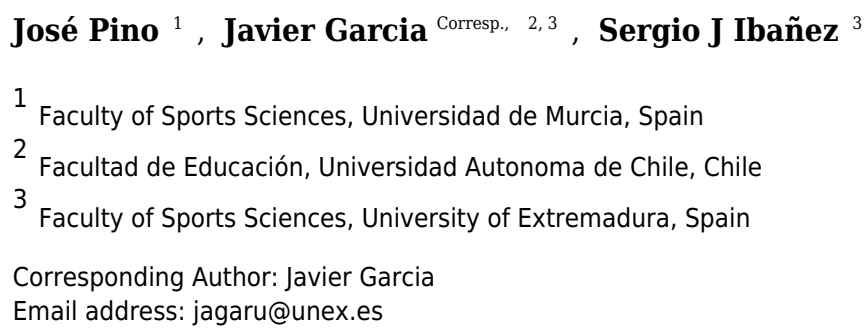

The aim of this study was to test the validity and reliability of the inertial device WIMU (Realtrack Systems SL, SPA) for the assessment of the vertical jump, counter movement jump and squat jump. Fifteen soccer players were evaluated in two identical sessions separated by one week. In each session, participants performed three jumps of each type. The flight time was quantified by the inertial device WIMU and by a force platform (Twin plates, Globus) at the same time. For the analysis of reliability of the flight time of the CMJ and the SJ, the intraclass correlation coefficient (ICC) was used. The calculation of the concurrent validity was performed by using the Pearson correlation coefficient ( $r$ ). This analysis was complemented with the realization of the Bland-Altman plots. For the analysis of reliability, the coefficient of variation and the standard error of the means were calculated. The analysis presented a high validity and reliability of the device. The results show the inertial device WIMU (Realtrack Systems SL, SPA) as a useful tool for measuring the jump capacity of the athletes, presenting immediate results in real time, on any type of surface and in a simple way since it does not need cables. 
1

2

3 BACKGROUND: The aim of this study was to test the validity and reliability of the inertial device

4 WIMU $^{\mathrm{TM}}$ (Realtrack Systems SL, SPA) for the assessment of different vertical jump modalities.

5 METHODS: Fifteen soccer players were evaluated into two identical sessions separated by one

6 week. In each session, participants performed three jumps of each type. The flight time was

7 quantified by the inertial device WIMU ${ }^{\mathrm{TM}}$ and by a force plate (Twin plates, Globus) at the same

8 time. The intraclass correlation coefficient (ICC) was used for the analysis of reliability of the

9 flight time of the Counter Movement Jump and the Squat Jump. For the analysis of reliability, the

10 coefficient of variation and the standard error of the means were calculated. The calculation of the

11 concurrent validity was performed by using the Pearson correlation coefficient (r). This analysis

12 was complemented with the realization of Bland-Altman plots.

13 RESULTS: The analysis presented a high validity $(\mathrm{CMJ}$ : $\mathrm{ICC}=0.97, r=0.95$; $\mathrm{SJ}$ : ICC $=0.96, r$ :

14 0.93) and reliability (CMJ: $\mathrm{CV}=3.1 ; \mathrm{SJ}: r=2.5)$ of the device.

\section{ABSTRACT}

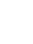




\section{INTRODUCTION}

One of the most commonly used indicators to assess physical fitness in different in both the general population and high performance sports, and for identifying young talents (1) is the vertical jump. Similarly, it has been used to predict the risk of injury and is associated with muscle power or neuromuscular fatigue $(2,3)$. In fact, jump performance plays a crucial role in both the predominantly anaerobic and aerobic disciplines, as well as in the mixed disciplines $(4,5)$. This can be measured by different tools or systems (force plates, video analysis, photoelectric cells, contact mat, video cameras, etc.) (6-8) and more recently with phone apps as MyJump (1). Although they are highly accurate measurement systems, they involve some drawbacks. For example, force plates are difficult to use in field test while the video analysis does not generate immediate results (1). Similarly, video analysis has a major drawback, especially if only one camera is used. If the plane that records the camera moves, the accuracy will be affected (9). There are several means for evaluating vertical jump, therefore it is important to choose the one that bestfits to complete the task, regarding precision, cost, reliability or duration (6). Accelerometers, however, are a highly portable, lightweight, easy to use and accessible tool for almost any coach $(7,10)$.

The vertical jump height is measured as the difference between the position of the center of mass of the individual in the starting position (a standing position) and its position at the maximum height (11). This height can be measured according to the flight time (12). Flight time is the raw data that both devices recorded and is used in several studies (13-15) and reported as the more accurate method to calculate jump height (16). The estimation of jumping performance is done after the event. Also, the jump height is an indirect technique to measure jump height or muscle power and as (8) suggest, the different methods used in the calculation could be led to a systematic 
42 error. Not all the measurement systems are equally accurate, in fact it has been reported that, due

43 to the different angles of the knees and ankles at the time of the jump starts, there may be an error

44 of $2.2 \mathrm{~cm}(15)$. WIMU ${ }^{\mathrm{TM}}$ is an inertial device designed for monitoring the physical activity for

45 athletes of different disciplines.

46 The control of the validity and reliability of the WIMU ${ }^{\mathrm{TM}}$ device is necessary for its acceptance as

47 an accurate measurement system for assessing the vertical jump. The validity and reliability refer

48 to the device's ability to measure what it is designed to measure (validity), and to always measure

49 the same event inthe same way (reliability) (17). Therefore, the objective of this study was to

50 evaluate the validity and reliability of the WIMU ${ }^{\mathrm{TM}}$ device for assessing SJ and CMJ. It has been

51 hypothesized that the WIMU ${ }^{\mathrm{TM}}$ device will display a high validity and reliability for measuring

52 the different vertical jumps used in the study.

\section{MATERIALS \& METHODS}

\section{Experimental approach to the problem}

55 The aim of this study was to test the validity and reliability of the device WIMU (Realtrack

56 Systems SL, SPA) to measure the performance in the vertical jump. In order to do that, the tests

57 for measuring the flight time during a slow cycle of stretching and shortening the muscle (CMJ)

58 and the explosive concentric muscle actions (SJ) were conducted by 15 subjects into two identical

59 sessions. Data collections were separated by one week. Jumps were measured with the WIMUTM

60 device and the force plate at the same time (Twin Plates, Globus Sport and Health Technologies

61 LLC, ITA), considered as the gold standard. The election of flight time as variable of comparison

62 is because is the direct data that provide both systems. Jump height is an indirect measurement of 
63 a formula based on flight time. WIMU ${ }^{\mathrm{TM}}$ was attached to a belt and fixed on the lower back

64 (Choukuo et al., 2014).

65

66

\section{Subjects}

The participants in the study were 15 soccer players at early stages $(\mathrm{N}=15$, age $=14.74 \pm 0.23$ years old, height $=164.34 \pm 4.04 \mathrm{~cm}$, weight $=65.7 \pm 3.35 \mathrm{~kg})$. All the participants belonged to the same soccer team participating at a regional category in Spain. The team trains three sessions of two hours per week, with a total volume of six hours of training. The jumps were performed during the competitive season. All subjects and their parents were informed about the study procedures and their possible risks, giving their consent to participate before testing. The ethics committee of the University approved the study ( $\left.n^{\circ} 67 / 2017\right)$.

\section{Procedure.}

In both sessions of data acquisition, conditions were the same. During the week of training, the jumps were performed three days after the last game. Before jumping, warming-up was equal in both sessions. A standard warm-up of 15 minutes, consisting of five minutes of continuous running, specific mobility of the lower body for another 5 minutes, active stretching of the lower extremities, and vertical jumps for the last 5 minutes $(1,7)$. Previously, the subjects had been instructed in performing the different jumps by the same examiner.

Testing procedures: The subjects performed each CMJ starting from a static standing position, with their arms on their hips and with their knees extended during the flight. Once in position, the subjects were instructed (approximately a $90^{\circ}$ angle) as quickly as possible and then jump as high as possible. In SJ, subjects began in the same standing position as in the CMJ, also with their hands on their hips. In this position, they were asked to flex their hips (aprox. $90^{\circ}$ ) and maintain this 
85 position. After that, the examiner made them wait for three seconds and then cheered them up

86

87

verbally to jump as high as possible, without making any type of counter movement. In all jumps at takeoff, participants were asked to leave the floor at the same time and always with their knees extended, and land in similarly extended position $(7,18)$. A total of six jumps were evaluated for each participant, measured with the inertial device WIMU and the contact plate (figure 1).

$W I M U^{T M}$ (Figure 2). For this study, trials have been recorded by the accelerometer at a sampling frequency of $1000 \mathrm{~Hz}$. Accelerometers and gyroscopes integrated in the device were used to correct and calculate the vertical acceleration recordings. All the information is represented in its specific software QÜIKO ${ }^{\mathrm{TM}}$, which allows automatic analysis. A mathematic algorithm was developed in order to calculate flight time using total acceleration signal (vector sum of three axis of the accelerometer and information recorded by gyroscopes). Jump height can be calculated with Bosco, Luhtanen (12) equation.

Force Plate. The force plate "Twin Plates" (Globus Sport and Health Technologies LLC, ITA) $(240 \times 400 \mathrm{~mm})$ records the data at a frequency of $1000 \mathrm{~Hz}$ with an error of less than $1 \%$. It was used at the same time as the inertial device WIMU ${ }^{\mathrm{TM}}$. The plate was connected to a laptop computer for the real-time feedback via the software "Ergo system" (Globus Sport and Health Technologies LLC, ITA). Flight time is automatic calculated by the force platform software.

\section{Statistical analysis}

A first descriptive analysis with averages and standard deviation was performed to characterize the sample. The intraclass correlation coefficient (ICC) (2.1) was used for the analysis of reliability of the flight time of the CMJ and the SJ. The Coefficient of Variation (CV) was used to analyze the reliability of the instrument. The absolute reliability was determined by calculating the indexes 
107 Standard Error of Measurement (SEM) $[\mathrm{SEM}=\mathrm{SD} \sqrt{ }(1-\mathrm{ICC})$ where $\mathrm{SD}$ is the standard deviation

108 of day one and day two] and the Minimum Real Change (SRD) [SRD=1.96 x $\sqrt{2} \times \mathrm{SEM}]$ (17). Both

109 SEM and the SRD were calculated in absolute terms and in percentage for easier interpretation

110 and comparison with other measuring devices. Similarly, both the SEM and the SRD became

111 percentages to facilitate comparison with other studies. The percentages were calculated according

112 to the following equation: $\mathrm{SEM} \%=(\mathrm{SEM} / \mathrm{mean}$ flight time of the two repetitions $) \cdot 100 ;$ and

$113 \mathrm{SRD} \%=(\mathrm{SRD} / \mathrm{mean}$ flight time of the two repetitions $) \cdot 100$. All the reliability tests were performed

114 for the force plate and the inertial device. Similarly, the SEM and the SRD are indicators that 115 express the absolute reliability of the device, shown at the same measurement unit of the 116 instrument. Besides, the SEM results are highly independent from the population under study, not

117 as the ICC (17). To extrapolate the results of the study, it has been chosen to express these indexes

118 in absolute terms, thus being able to compare the results with those of other instruments more 119 easily (19).

120 The calculation of the concurrent validity was performed by using the Pearson correlation 121 coefficient (r). This analysis was complemented with the realization of the Bland-Altman plots.

122 This representation indicates the degree of agreement between both instruments, not only the 123 degree of relation (20).

124 The level of significance was established at $\mathrm{P}<0.05$. All of the analyses were performed by using 125 the statistical package SPSS 21.0 for Windows (IBM Co., USA), except for the Bland-Altman 126 plots, which were performed by using the software Graphpad Prism (Graphpad, Inc., USA).

\section{RESULTS}


128 The descriptive analysis showed that the flight time registered by the inertial device WIMU ${ }^{\mathrm{TM}}{ }_{\text {Was }}$

129 almost identical than the one registered by the plate (Table 1), but there were no significant

130 differences between both devices. The average flight time in the CMJ was: force plate $=437.62$

$131 \mathrm{~ms}$; WIMU ${ }^{\mathrm{TM}}$ device $=437.31 \mathrm{~ms}$. For the SJ, flight time was: force plate $=416.72 \mathrm{~ms}$; WIMUTM

132 device $=416.11 \mathrm{~ms}$. The results showed an almost perfect relation between the inertial device

133 WIMU ${ }^{\mathrm{TM}}$ and the contact platform, both in the CMJ (ICC $(2.1)=0.97,95 \%$ CI: $0.96-0.98, \mathrm{P}<$

$1340.001)$ as in the SJ $(\operatorname{ICC}(2.1)=0.96,95 \%$ CI: $0.94-0.97, \mathrm{P}<0.001)$. The reliability analysis

135 showed a SEM\% of $2.2 \%$ for the CMJ and of $1.4 \%$ for the SJ. The SRD $\%$ is $6.2 \%$ for the CMJ

136 and $3.9 \%$ for the SJ. The CVs were very low for both periods of flight time of jump (CMJ: $3.1 \%$;

137 SJ: 2.5\%) (Table 1). In addition, the Pearson correlation coefficient is almost perfect in both cases

138 ( $\mathrm{r}>.9)$ (Table 1 and Figure 3 and 4).

139 Bland-Altman plots show an average systematic trend of $1.31(0.29 \%)$ milliseconds between the 140 force plate and the inertial device in the CMJ; and an average systematic trend of $0.61(0.13 \%)$ in

141 the SJ. The trend is the average difference between the two measures. As these values are positive, 142 the force plate gets higher values than the inertial device (Figures 5 and 6).

\section{DISCUSSION}

144 The objective of the present study was to evaluate the validity and reliability of the WIMUTM 145 device for the assessment of the flight time of squat jump and countermovement jump. The results 146 show the high concurrent validity and reliability that the WIMU ${ }^{\mathrm{TM}}$ device presents compared with 147 a force plate. The WIMU ${ }^{\mathrm{TM}}$ device has proven to be a useful instrument. The results from this 148 study conclude that WIMU ${ }^{\mathrm{TM}}$ provides similar flight times in squat jump and countermovement 149 jump to the criterion method. 
150 In previous studies, correlation coefficient has been the main method to assess validity and

151 reliability. It has been suggested that other methods, as Bland-Altman plots, provide more relevant

152 information about agreement between two measures (20). For example, it has been probed that

153 Myotest and Optojump have a high correlation according to ICCs (0.98), but with a systematic

154 bias of around $7 \mathrm{~cm}$ in vertical jump (7). In fact, a constant error will not be detected on the

155 correlation analysis and, therefore, it cannot be concluded that devices are accurate (9). The data

156 presented in the Bland-Altman plots show that most of the jumps are close to the media of the

157 differences between instruments in both jump modalities, showing a high level of agreement (20)

158 and a high correlation between them. The ICC presents a great precision of measurements $(>0.93)$.

159 Similarly, by analyzing the reliability of the devices, excellent CVs $(<10 \%)$ can be appreciated

160 (11, 19). The SEM\% shows very low percentages of absolute error $(21)$.

161 Several studies have examined the validity of different methods of analysis of the vertical jump in 162 comparison to force plates. Previous studies have misreported the vertical jump performance,

163 found discrepancies between devices compared with force plates of around $10 \mathrm{~cm}$ measuring flight 164 time $(16,22)$ or measuring jump height, from recorded flight time, in contact mats $(16,23)$. It has 165 also been reported that mastery of the jumping technique may affect the jump performance (23).

166 On the other hand, contact mats measure flight time according to the moment when the subject 167 leaves the ground, and miss some data of the initial rise of the centre of mass before the take-off 168 (23). Also, the differences could be due to the different ascending and descending phases and 169 landing (16). In fact, some studies report as limitation the lack of gyroscope that can detect the 170 inclination of the body in take-off and landing $(7,25)$.

171 Studies have shown lower values for the validity (ICCs scores), of their devices to those found 172 with the WIMU ${ }^{\mathrm{TM}}$. Choukou, Laffaye (11) studies the validity of the Myotest in comparison to a 
173 force plate, finding ICC values between 0.86-0.96. Casartelli, Müller (7), by studying the same

174 device, finds similar results. The analysis of reliability shows very low CVs, better than those

175 found when the reliability of mobile applications was developed (26), photoelectric cells (27), or

176 other inertial devices such as accelerometers $(7,11)$. When the results are compared to the ones

177 obtained with high-speed cameras, the results are practically the same (25). Force plates are

178 considered the most accurate tools for measuring the vertical jump, since they allow identifying

179 the moment of take-off with great precision $(25,27,28)$. This device, force platform, is enable to

180 measure, in eccentric and concentric phase of the movement, the force and power production (23),

181 allowing more detailed analysis of subjects' training. WIMU ${ }^{\mathrm{TM}}$ allows providing a similar analysis

182 of force plates (as results concluded) and, contrary to force plates, WIMU ${ }^{\mathrm{TM}}$ still records data of

183 more variables during flight time (such as $\mathrm{G}$ force in take-off and landing or inclination, among

184 others), which makes the analysis more detailed. These results demonstrate the ability of the

185 WIMU ${ }^{\mathrm{TM}}$ system to play the measurements that a force plate makes, having the advantage of being

186 lightweight and portable. These measurements are valid for both the CMJ and the SJ. Due to its

187 properties of size and weight, this device can be easily placed in any segment of the body to

188 measure the vertical jump (center of mass, hips, back, lower body, etc.). The data collection is

189 done with a single computer wirelessly connected to the device, so a great time preparing for the

190 test is not needed. In addition, subjects do not have to be connected by any cable or have to take

191 off and land in a delimited area. Moreover, thanks to the specific software, the device provides

192 immediate feedback.

\section{CONCLUSIONS}

194 Because of the ability of this device to collect data on different types of jump and the relevant 195 information from these, WIMU ${ }^{\mathrm{TM}}$ is a valuable tool for controlling the training and competition. 
196 Throughout control of the jumps, changes produced by a particular training program can be

197 detected, being able to redirect or modify it according to the results (7). It can also be used to

198 determine the fatigue accumulated during the same work, adapting the breaks between sets for a

199 full recovery $(10,29)$. The WIMU ${ }^{\mathrm{TM}}$ device does not need cables, so it greatly facilitates its

200 placement in the subject's body as well as the freedom of movement.

201

202

203

204

205

206

207

208

209

210

211

212

213

214

215

216

217

218

219

220

221

222

223

224

225

226

227

228

229

230

231

232

233

234

235

236

\section{REFERENCES.}

1. Balsalobre-Fernández C, Glaister M, Lockey RA. The validity and reliability of an iPhone app for measuring vertical jump performance. Journal of sports sciences. 2015;33(15):1574-1579.

2. Jimenez-Reyes P, Pareja-Blanco F, Rodriguez-Rosell D, Marques MC, Gonzalez-Badillo JJ. Maximal Velocity as a Discriminating Factor in the Performance of Loaded Squat Jumps. International journal of sports physiology and performance. 2016;11(2):227-234.

3. Stevenson JH, Beattie CS, Schwartz JB, Busconi BD. Assessing the Effectiveness of Neuromuscular Training Programs in Reducing the Incidence of Anterior Cruciate Ligament Injuries in Female Athletes $A$ Systematic Review. The American journal of sports medicine. 2015;43(2):482-490.

4. Brumitt J, Heiderscheit BC, Manske RC, Niemuth PE, Rauh MJ. Off-season training habits and preseason functional test measures of division iii collegiate athletes: a descriptive report. International journal of sports physical therapy. 2014;9(4):447-455.

5. Hartman MJ, Clark B, Bemben DA, Kilgore JL, Bemben MG. Comparisons between twice-daily and once-daily training sessions in male weight lifters. International journal of sports physiology and performance. 2007;2(2):159-169.

6. Bui HT, Farinas MI, Fortin AM, Comtois AS, Leone M. Comparison and analysis of three different methods to evaluate vertical jump height. Clinical physiology and functional imaging. 2015;35(3):203209.

7. Casartelli N, Müller R, Maffiuletti NA. Validity and reliability of the myotest accelerometric system for the assessment of vertical jump height. The Journal of Strength \& Conditioning Research. 2010;24(11):3186-3193.

8. Dias JA, Dal Pupo J, Reis DC, Borges L, Santos SG, Moro AR,Borges Jr NG. Validity of two methods for estimation of vertical jump height. The Journal of Strength \& Conditioning Research. 2011;25(7):2034-2039.

9. Magnúsdóttir Á, Karlsson B. Comparing three devices for jump height measurement in a heterogeneous group of subjects. The Journal of Strength \& Conditioning Research. 2014;28(10):28372844.

10. Sato K, Smith SL, Sands WA. Validation of an accelerometer for measuring sport performance. The Journal of Strength \& Conditioning Research. 2009;23(1):341-347.

11. Choukou M-A, Laffaye G, Taiar R. Reliability and validity of an accelerometric system for assessing vertical jumping performance. Biology of sport. 2014;31(1):55-62.

12. Bosco C, Luhtanen P, Komi PV. A simple method for measurement of mechanical power in jumping. European journal of applied physiology and occupational physiology. 1983;50(2):273-282.

13. Balsalobre-Fernández C, Tejero-González CM, del Campo-Vecino J, Bavaresco N. The concurrent validity and reliability of a low-cost, high-speed camera-based method for measuring the flight time of vertical jumps. The Journal of Strength \& Conditioning Research. 2014;28(2):528-533. 
237 14. García-López J, Morante JC, Ogueta-Alday A, Rodríguez-Marroyo JA. The type of mat (Contact vs.

238 Photocell) affects vertical jump height estimated from flight time. The Journal of Strength \&

239 Conditioning Research. 2013;27(4):1162-1167.

240 15. Garcia-Lopez J, Peleteiro J, Rodgriguez-Marroyo J, Morante J, Herrero J, Villa J. The validation of

241 a new method that measures contact and flight times during vertical jump. International journal of

242 sports medicine. 2005;26(4):294-302.

243 16. Aragón LF. Evaluation of four vertical jump tests: Methodology, reliability, validity, and accuracy.

244 Measurement in physical education and exercise science. 2000;4(4):215-228.

245 17. Weir JP. Quantifying test-retest reliability using the intraclass correlation coefficient and the 246 SEM. Journal of strength and conditioning research. 2005;19(1):231-240.

247 18. Häkkinen K, Komi PV. Effect of explosive type strength training on electromyographic and force

248 production characteristics of leg extensors muscles during concentric and various stretch-shortening

249 cycle exercises. Scand J Sports Sci. 1985;7(2):65-76.

250 19. Atkinson G, Nevill AM. Statistical methods for assessing measurement error (reliability) in

251 variables relevant to sports medicine. Sports medicine. 1998;26(4):217-238.

252 20. Bland JM, Altman D. Statistical methods for assessing agreement between two methods of

253 clinical measurement. The lancet. 1986;327(8476):307-310.

254 21. Collado-Mateo D, Adsuar JC, Olivares PR, Cano-Plasencia R, Gusi N. Using a dry electrode EEG

255 device during balance tasks in healthy young-adult males: Test-retest reliability analysis. Somatosensory

256 \& motor research. 2015;32(4):219-226.

257 22. Moir GL. Three different methods of calculating vertical jump height from force platform data in

258 men and women. Measurement in Physical Education and Exercise Science. 2008;12(4):207-218.

259 23. Buckthorpe M, Morris J, Folland JP. Validity of vertical jump measurement devices. Journal of 260 sports sciences. 2012;30(1):63-69.

261 24. Leard JS, Cirillo MA, Katsnelson E, Kimiatek DA, Miller TW, Trebincevic K, Garbalosa J. Validity of

262 two alternative systems for measuring vertical jump height. The Journal of Strength \& Conditioning

263 Research. 2007;21(4):1296-1299.

264 25. Requena B, García I, Requena F, de Villarreal ES-S, Pääsuke M. Reliability and validity of a

265 wireless microelectromechanicals based system (Keimove ${ }^{\mathrm{TM}}$ ) for measuring vertical jumping

266 performance. Journal of sports science \& medicine. 2012;11(1):115-122.

267 26. Gallardo-Fuentes F, Gallardo-Fuentes J, Ramírez-Campillo R, Balsalobre-Fernández C, Martínez C,

268 Caniuqueo A, Cañas R, Banzer W, Loturco I, Nakamura F, Izquierdo M. Inter And Intra-Session Reliability

269 And Validity Of The My Jump App For Measuring Different Jump Actions In Trained Male And Female

270 Athletes. The Journal of Strength \& Conditioning Research. 2015;30(7):2049-2056.

271 27. Glatthorn JF, Gouge S, Nussbaumer S, Stauffacher S, Impellizzeri FM, Maffiuletti NA. Validity and

272 Reliability of Optojump Photoelectric Cells for Estimating Vertical Jump Height. The Journal of Strength

273 \& Conditioning Research. 2011;25(2):556-560.

274 28. Enoksen E, Tønnessen E, Shalfawi S. Validity and reliability of the Newtest Powertimer 300-

275 series $^{\circledR}$ testing system. Journal of Sports Sciences. 2009;27(1):77-84.

276 29. Haff GG, Whitley A, Mccoy LB, O'Bryant HS, Kilgore JL, Haff EE, Pierce K, Stone MH. Effects of

277 different set configurations on barbell velocity and displacement during a clean pull. The Journal of

278 Strength \& Conditioning Research. 2003;17(1):95-103.

279 Figure 1. A graphic example of flight time data of WIMU and force Plate.

280 Figure 2. WIMU inertial device.

Figure 3. Concurrent validity between force plate and WIMU: CMJ. 
282 Figure 4. Concurrent validity between force plate and WIMU: SJ.

283 Figure 5. Bland-Altman plots for force plate and WIMU in SJ. The central line represents the absolute average difference between 284 instruments. Short-dashed lines represent the upper and lower 95\% limits of agreement.

285 Figure 6. Bland-Altman plots for force plate and WIMU in CMJ. The central line represents the absolute average difference between instruments. Short-dashed lines represent the upper and lower $95 \%$ limits of agreement. 


\section{Figure 1}

A graphic example of flight time data of WIMU and force plate.

Squat jump recorded by WIMU and Force platform.

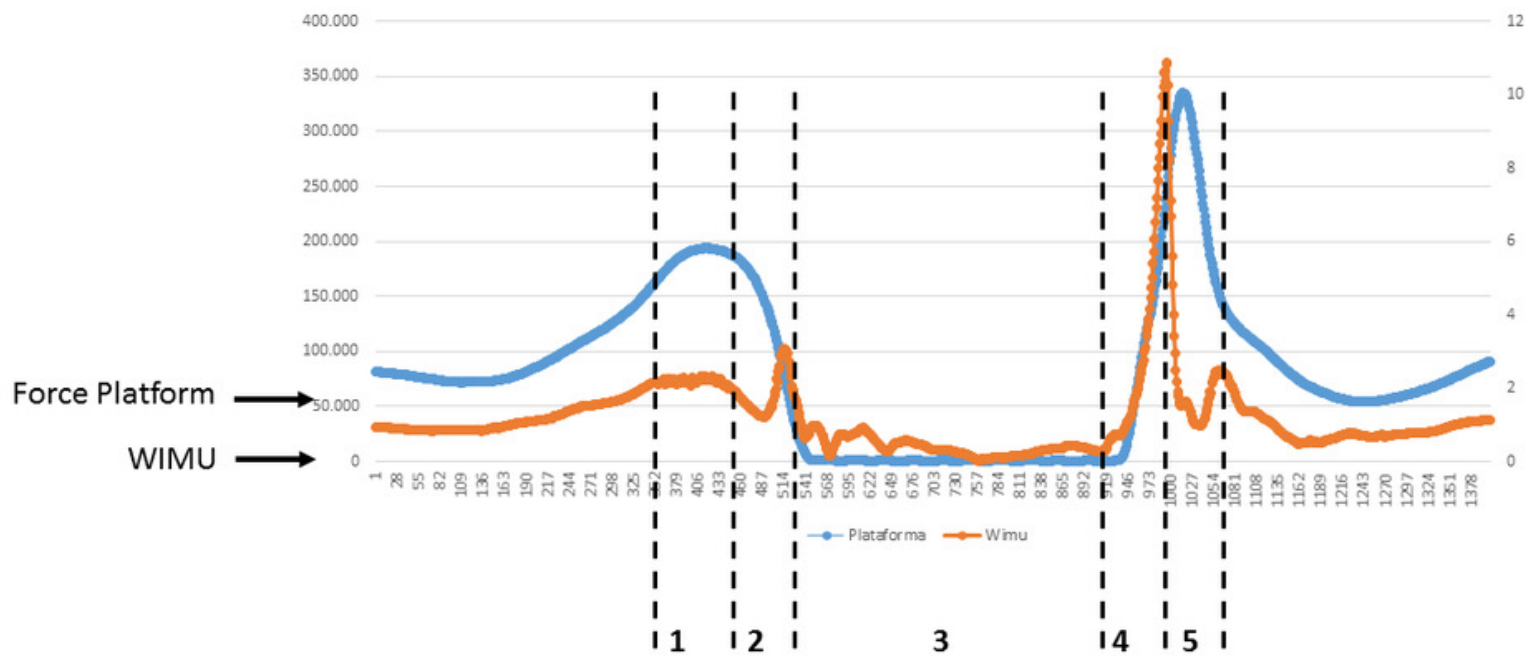

(1) Knees flexion (concentric); (2) Takeoff; (3) Flight time; (4) Touch-down; (5) Knees flexion (eccentric). The orange line represent Wimu inertial device measured in gs (right axis). Blue line represent force platform measured in $\mathrm{Ns}$ (Left axis) 


\section{Figure 2}

WIMU inertial device

Photo credit: Sergio Ibáñez and Javier García.

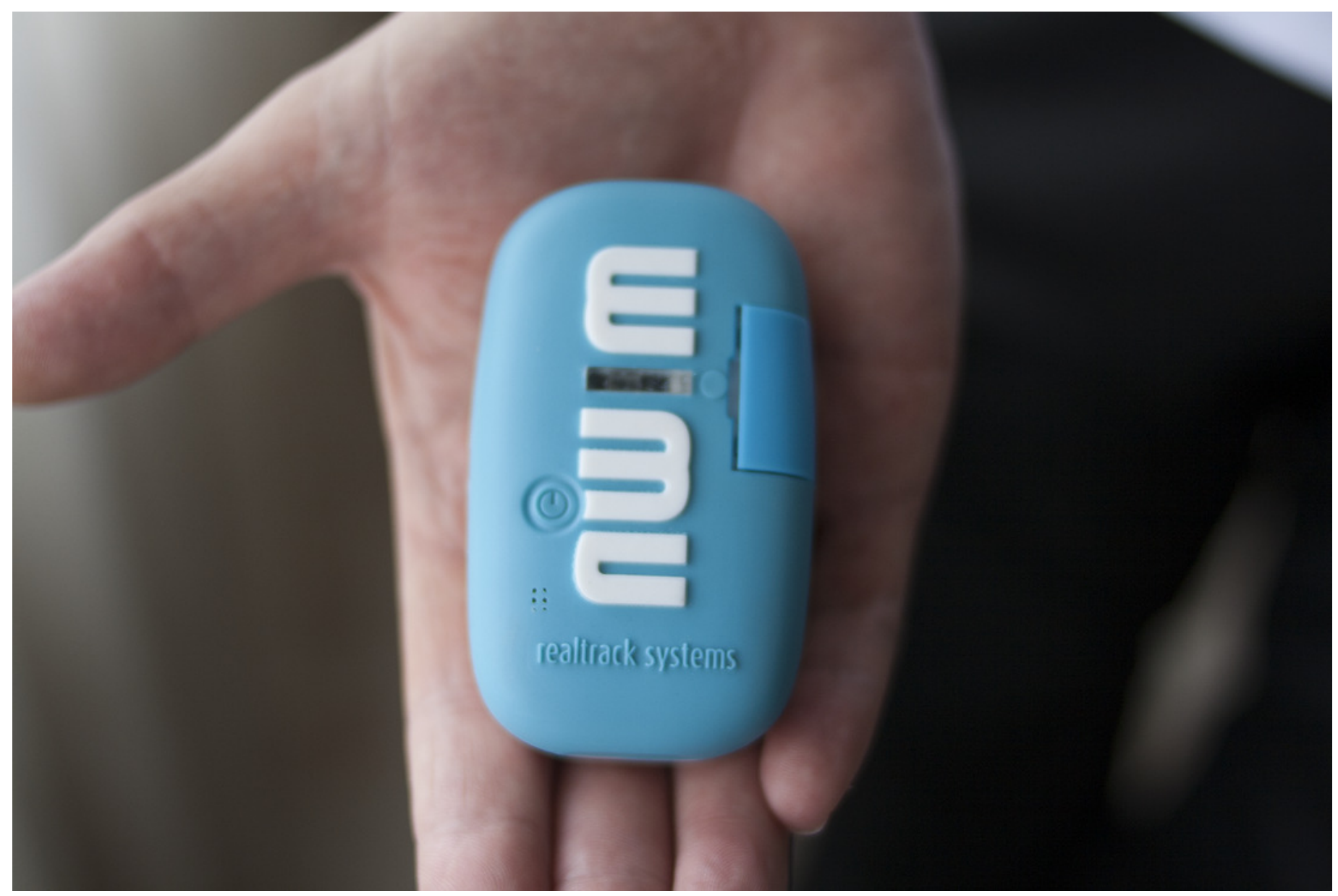


Figure 3

Concurrent validity between force plate and WIMU: CMJ.

Correlation of CMJ (ms).

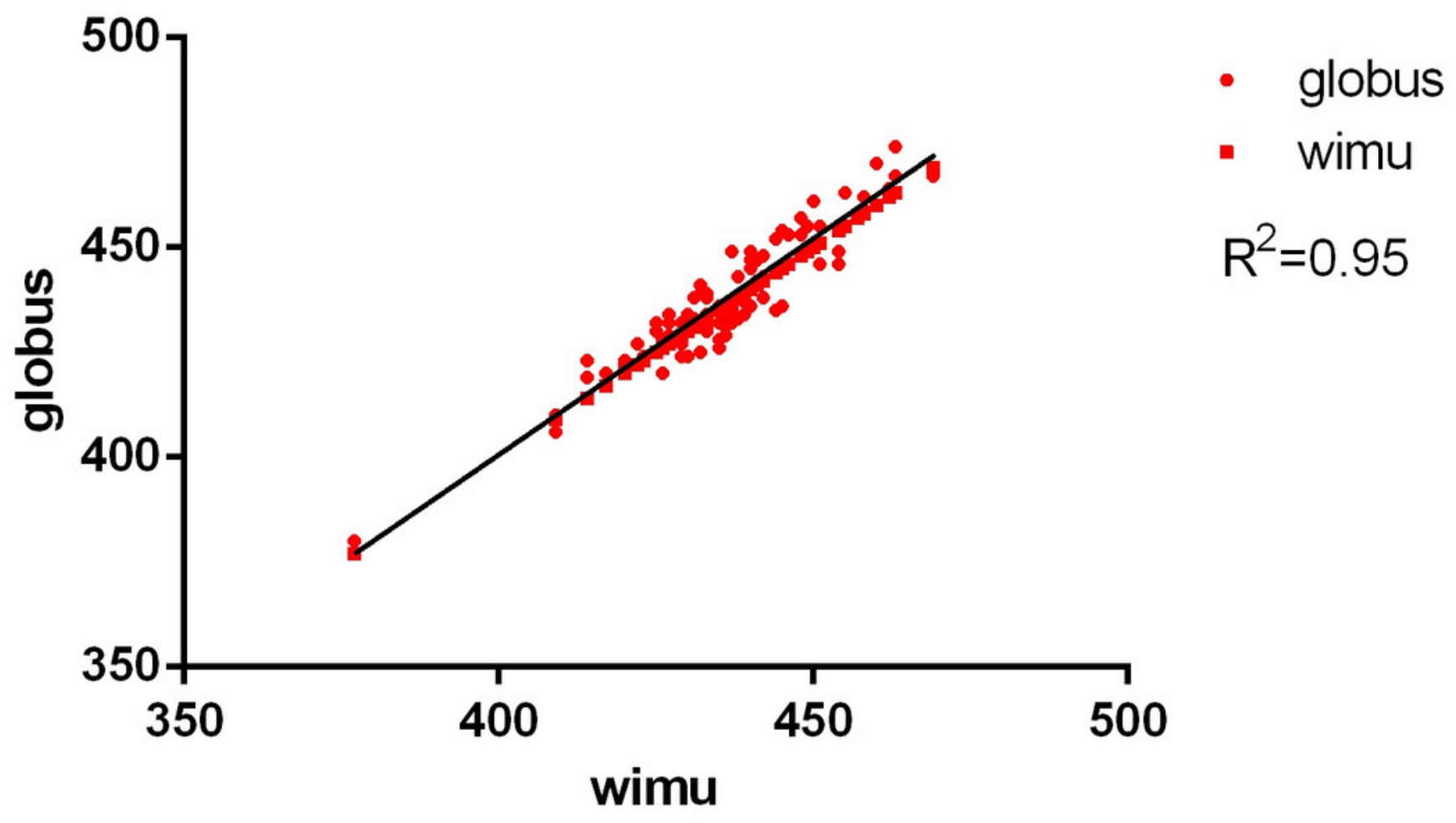


Figure 4

Concurrent validity between force plate and WIMU: SJ.

Correlation of SJ (ms).

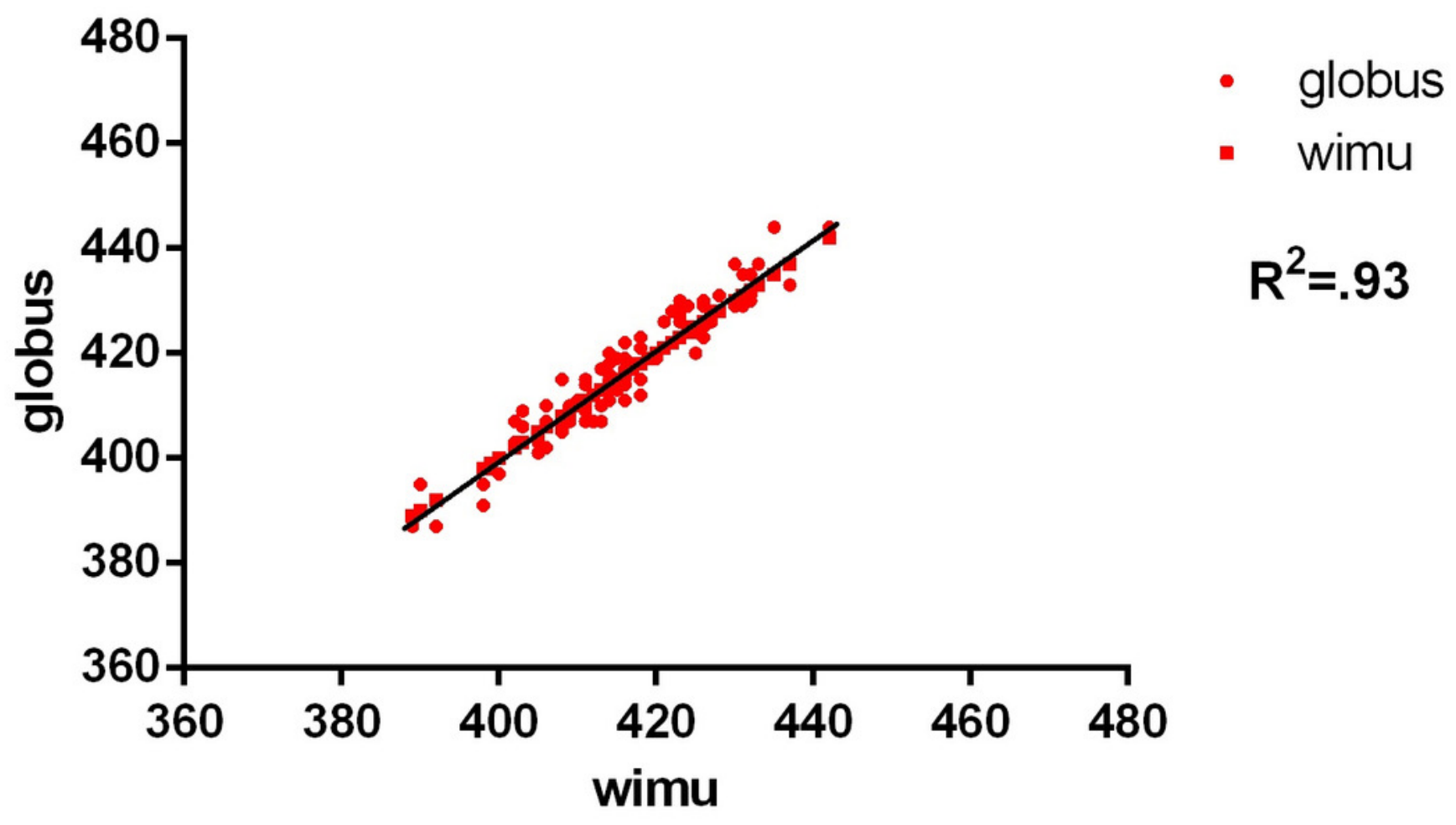


Figure 5

Bland-Altman plots for force plate and WIMU in SJ.

The central line represents the absolute average difference between instruments. Shortdashed lines represent the upper and lower $95 \%$ limits of agreement. Counter Movement Jump (Flight time) (ms).

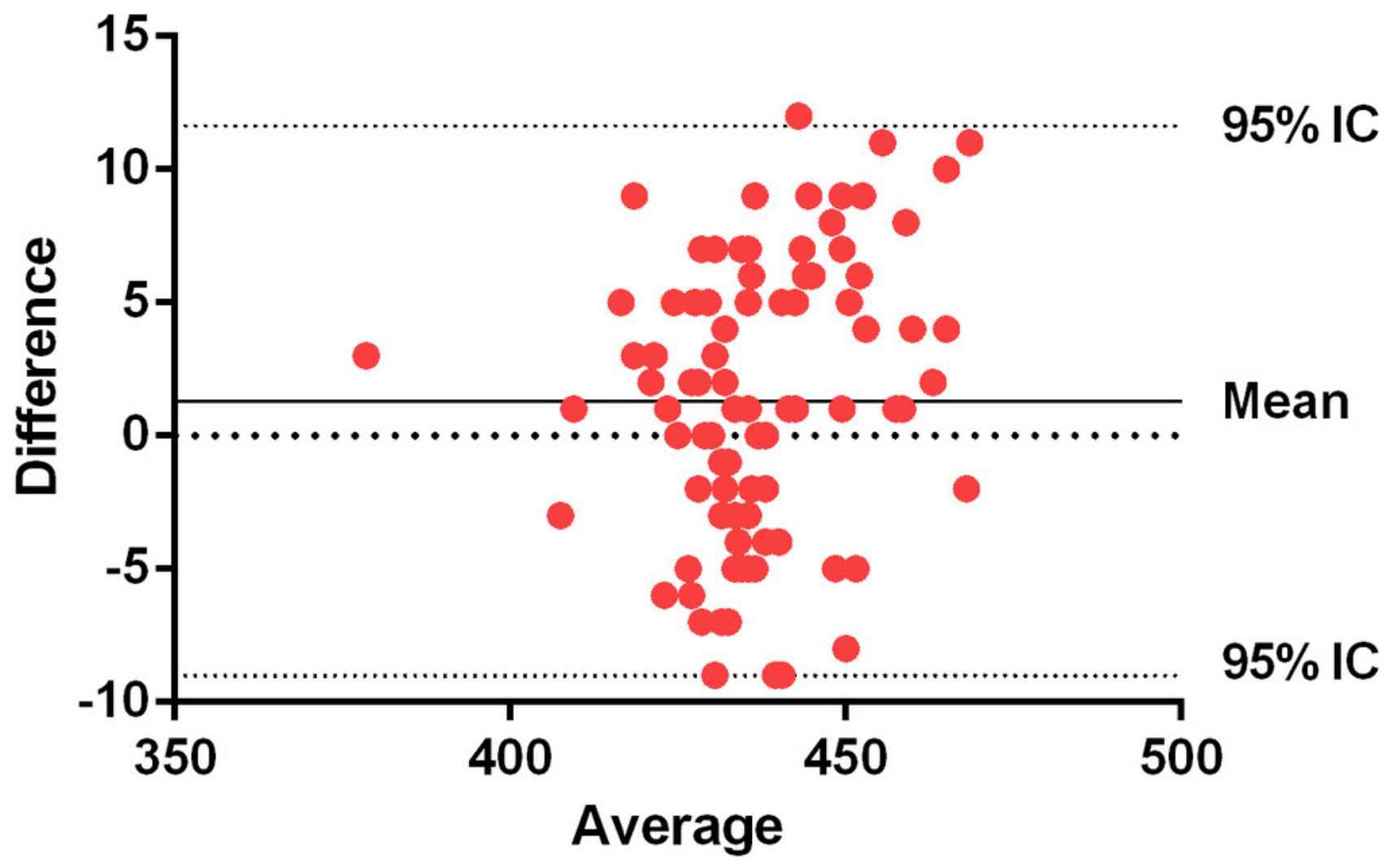


Figure 6

Bland-Altman plots for force plate and WIMU in CMJ.

The central line represents the absolute average difference between instruments. Shortdashed lines represent the upper and lower $95 \%$ limits of agreement. Squat Jump (Flight time) (ms).

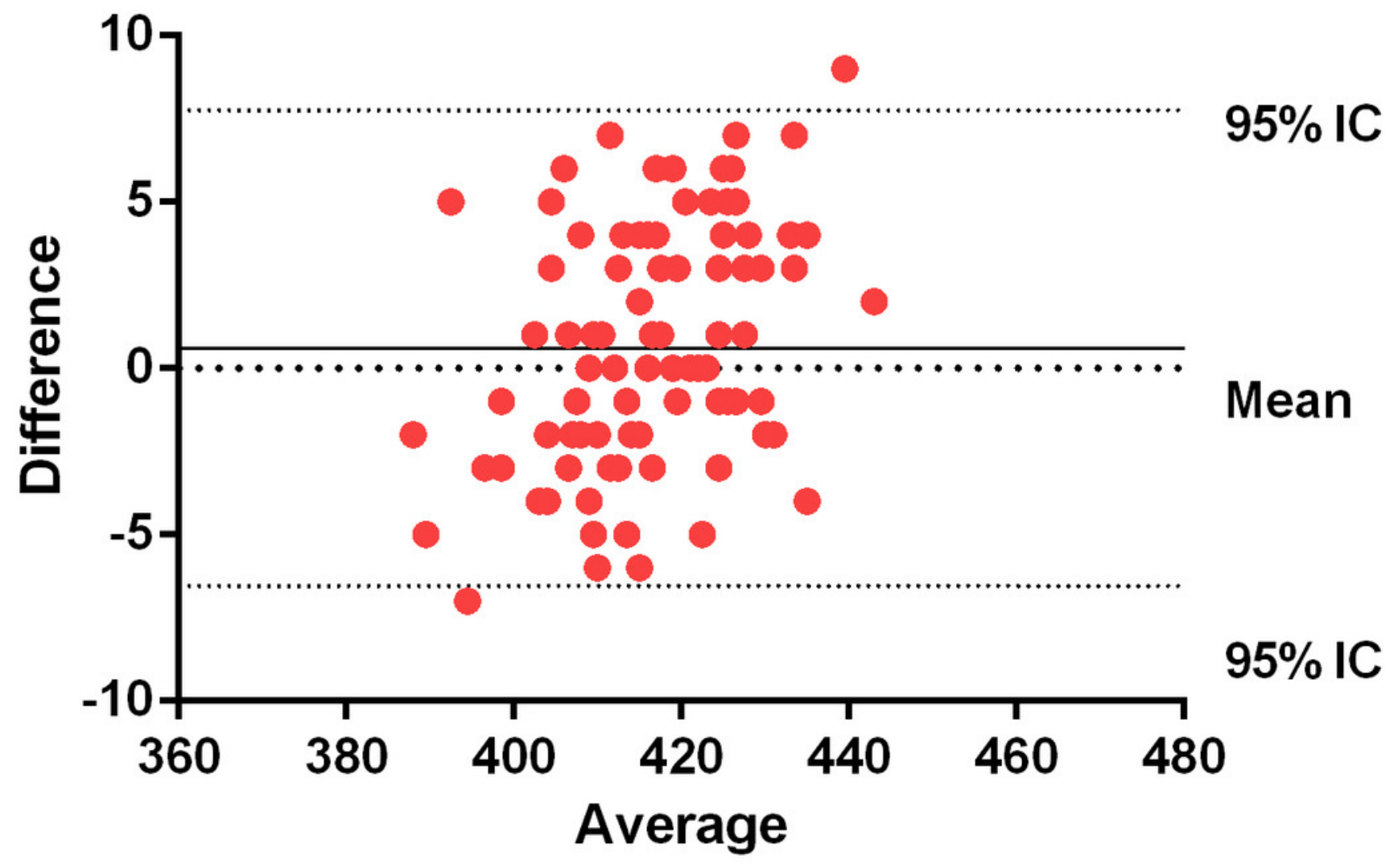




\section{Table $\mathbf{1}$ (on next page)}

Descriptive Statistics (Flight time in ms and jumping performance in $\mathrm{cm}$ ), intraclass correlation coefficients, reliability of the inertial device and flight time correlation of the CMJ and the SJ. 


\begin{tabular}{|c|c|c|c|c|c|c|c|c|c|c|c|}
\hline & & WIMU & & JCC & & & SEM & & SPD & & \\
\hline \multirow{3}{*}{ CMJ } & & & & \multirow{3}{*}{0.97} & \multirow{3}{*}{$0.96-0.98$} & \multirow{3}{*}{0.95} & \multirow{3}{*}{9.69} & \multirow{3}{*}{2.2} & \multirow{3}{*}{26.85} & \multirow{3}{*}{6.2} & \multirow{3}{*}{3.1} \\
\hline & Time & $436.31 \pm 13.70$ & $437.62 \pm 14.91$ & & & & & & & & \\
\hline & Performance & $23.34 \pm 0.02$ & $23.48 \pm 0.02$ & & & & & & & & \\
\hline \multirow[t]{2}{*}{ SJ } & Time & $416.11 \pm 10.70$ & $416.72 \pm 12.50$ & \multirow{2}{*}{0.96} & \multirow{2}{*}{$0.94-0.97$} & \multirow{2}{*}{0.93} & \multirow{2}{*}{5.60} & \multirow{2}{*}{1.4} & \multirow{2}{*}{16.35} & \multirow{2}{*}{3.9} & \multirow{2}{*}{2.5} \\
\hline & Performance & $21.23 \pm 0.01$ & $21.29 \pm 0.02$ & & & & & & & & \\
\hline
\end{tabular}

1 